\title{
False recollection of the role played by an actor in an event
}

\author{
Alan W. Kersten • Julie L. Earles • Christin Upshaw
}

Published online: 31 May 2013

(C) Psychonomic Society, Inc. 2013

\begin{abstract}
Two experiments demonstrated that eyewitnesses more frequently associate an actor with the actions of another person when those two people had appeared together in the same event, rather than in different events. This greater likelihood of binding an actor with the actions of another person from the same event was associated with high-confidence recognition judgments and "remember" responses in a remember-know task, suggesting that viewing an actor together with the actions of another person led participants to falsely recollect having seen that actor perform those actions. An analysis of age differences provided evidence that familiarity also contributed to false recognition independently of a false-recollection mechanism. In particular, older adults were more likely than young adults to falsely recognize a novel conjunction of a familiar actor and action, regardless of whether that actor and action were from the same or from different events. Older adults' elevated rate of false recognition was associated with intermediate confidence levels, suggesting that it stemmed from increased reliance on familiarity rather than from false recollection. The implications of these results are discussed for theories of conjunction errors in memory and of unconscious transference in eyewitness testimony.
\end{abstract}

Keywords Recollection · Familiarity in recognition memory $\cdot$ False memory $\cdot$ Aging

A professor at California State University-Hayward was attacked in front of his class of 141 undergraduates. Sworn

\footnotetext{
A. W. Kersten $(\bowtie)$

Department of Psychology, Florida Atlantic University, Boca Raton, FL 33431-0991, USA

e-mail: akersten@fau.edu

J. L. Earles $\cdot$ C. Upshaw

Wilkes Honors College, Florida Atlantic University, Boca Raton, FL, USA

testimony was collected from eyewitnesses immediately after the incident, and seven weeks later, the eyewitnesses were asked whether the perpetrator was present in a lineup of six pictures. Unbeknownst to the students at the time of the incident, however, the entire assault was staged, and thus investigators already knew who was the perpetrator. Only $40 \%$ of the eyewitnesses correctly identified the perpetrator. Perhaps more disturbingly, $25 \%$ of the eyewitnesses (including the professor himself) selected a photo of an innocent bystander who was simply present at the scene of the "crime" (Buckhout, 1974). This result exemplifies the phenomenon of unconscious transference, in which an eyewitness associates a familiar but innocent person with the actions of a criminal (Loftus, 1976).

Unconscious transference may occur for a number of reasons (Davis, Loftus, Vanous, \& Cucciare, 2008) under a variety of conditions, some of which may seldom be met in criminal cases (Read, Tollestrup, Hammersley, McFadzen, \& Christensen, 1990). The basic phenomenon of unconscious transference, however, closely resembles a robust finding in the memory literature, known as a conjunction memory error. Thus, theories of the mechanisms underlying conjunction memory errors may also explain at least some examples of unconscious transference that occur occasionally in criminal cases, and likely even more frequently in everyday life.

A conjunction memory error involves the false recognition of a test stimulus composed of features from two different study items. For example, Reinitz and Hannigan (2001) tested participants on face recognition. Some of the recognition lures combined features from two different faces within the study list. Participants were particularly likely to falsely recognize these conjunction faces when the features came from two faces that had been simultaneously present on the computer screen.

Reinitz and Hannigan $(2001,2004)$ explained this result in terms of a binding error in memory. In particular, the 
simultaneous presence of two faces on the computer screen may have resulted in both faces being represented in working memory, possibly leading to associations among the features of those faces. Thus, when participants were later presented with a conjunction of two such features, the retrieval of a previously established association may have caused them to recollect having seen those two features together in the same face. This same mechanism could also explain unconscious transference. In particular, if one considers people and the actions that they perform as being features of an event, then the simultaneous presence of an innocent bystander and the actions of a criminal may cause eyewitnesses to form associations between those two features, causing eyewitnesses to later recollect having seen the innocent bystander perform those actions.

An alternative explanation for conjunction memory errors came from Jones and Jacoby (2001). They proposed that conjunction errors occur because of the familiarity of the features of a stimulus in the absence of recollection of the contexts in which those features were encountered. In particular, the presence of two familiar features in a conjunction stimulus causes one to believe that one must have encountered this stimulus before, even though one has no explicit recollection of it. This theory could also explain unconscious transference. In particular, when considering the combination of the familiar face of a bystander and the familiar actions of a perpetrator, eyewitnesses may receive a strong feeling of familiarity, causing them to believe that the bystander must have committed the "crime," even though they could not explicitly recollect having seen the bystander perform these criminal actions.

The present research was designed to test the predictions of these two theories for conjunction errors in memory for events, using a method developed by Kersten, Earles, Curtayne, and Lane (2008; see also Earles, Kersten, Curtayne, \& Perle, 2008; Kersten \& Earles, 2010). This research was designed on the basis of the assumption that unconscious transference can be explained in terms of basic memory processes such as familiarity, recollection, and binding, and thus that it may not be necessary to expose research participants to emotionally laden criminal acts in order to observe unconscious transference. Participants were instead presented with a series of brief, everyday events, each involving an individual actor performing a simple action, such as cutting a string or stirring oatmeal. Participants were later tested on their recognition memory for these events. The critical test items involved a previously seen actor performing an action that had previously been performed by somebody else. Participants were more likely to falsely recognize these conjunction items than to falsely recognize events involving new actors and actions. Thus, participants remembered the actors and actions from the encoding events, but had difficulty remembering which actors performed which actions.
The age differences observed by Kersten et al. (2008) provided support for the theory of Jones and Jacoby (2001) as an explanation for conjunction memory errors. In particular, older adults were more likely than young adults to falsely recognize a novel conjunction of a familiar actor and action, even when the two age groups were equated on baseline memory performance. Confidence ratings following their recognition judgments indicated that older adults' greater rate of false recognition of the conjunction items was primarily associated with intermediate levels of confidence. This result suggests that older adults were making attributions on the basis of the familiarity of these items. If older adults had instead falsely recollected having seen the presented actor perform the presented action, as was suggested by the theory of Reinitz and Hannigan (2001), their greater rate of false recognition of the conjunction items would be expected to be associated with high confidence, given that prior research (e.g., Tulving, 1985; Yonelinas, 2001) has demonstrated that recollection is associated with high confidence ratings.

The age differences observed in these prior studies thus provide evidence for influences of familiarity in the absence of recollection as an explanation for conjunction errors in memory for events. It remains possible that false recollection might also be observed, however, if different stimuli were presented. Reinitz and Hannigan (2001) revealed that participants were particularly likely to falsely recognize a conjunction stimulus involving two features that were simultaneously present on the computer screen. Participants may thus be more likely to falsely associate an actor with the actions of another actor when both actors are simultaneously present.

To test this prediction, in the present research young and older adult participants were presented with events that each involved two people playing different roles. For example, as can be seen in Fig. 1, participants may have seen Actor 1 putting a jacket on Actor 2, Actor 3 painting Actor 4's fingernails, and Actor 5 holding a dust pan for Actor 6 . To test whether participants would falsely associate an actor with the actions of another, simultaneously present actor, two different types of conjunction items were later presented. Same-event conjunction items involved an actor performing an action that had previously been performed by a different actor within the same event. For example, as can be seen in Fig. 2, participants might now have seen Actor 2 putting a jacket on someone else. Thus, an actor appeared in the same event context in which she had appeared earlier, but now played the opposite role. Different-event conjunction items involved an actor performing an action that had previously been performed by an actor in a different event. For example, participants who saw the above stimuli might now have seen Actor 3 holding a dust pan for someone else. Thus, an actor appeared in a different event context at test than she had at encoding. 


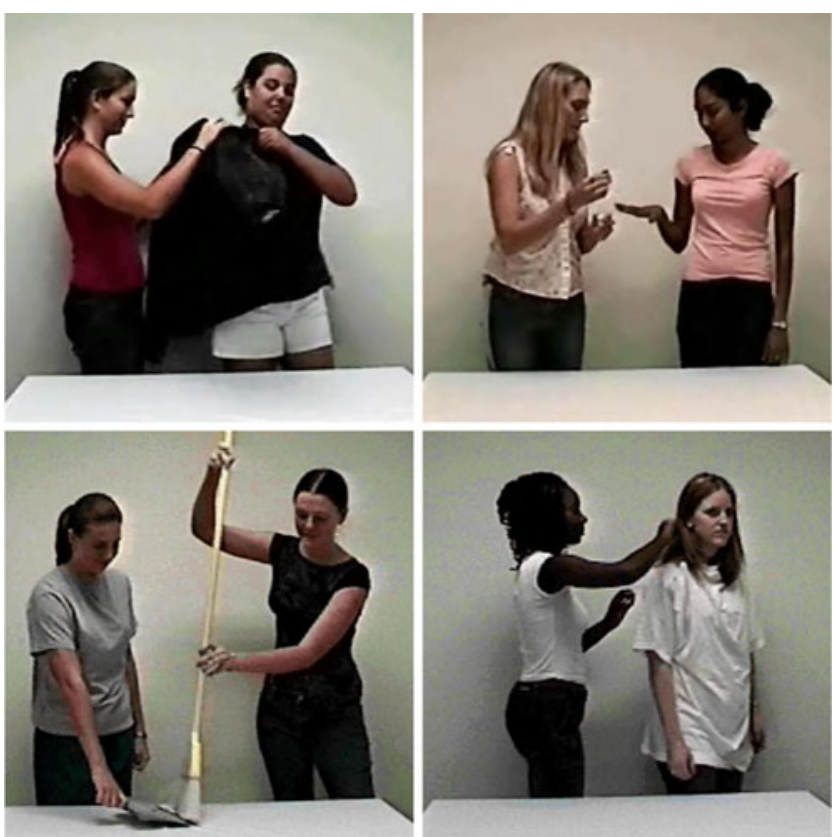

Fig. 1 Still frames from example encoding events

The two theories described above make different predictions for the present research. The theory of Reinitz and Hannigan (2001) suggests that seeing two actors perform two different actions within the same event could result in both actors and actions being simultaneously represented in working memory, potentially leading to incorrect associations between an actor and the actions of the other actor. Thus, this theory predicts that participants should be more likely to falsely recognize the same-event than the differentevent conjunction items. In particular, the combination of an actor and the actions of the other person from the same event might lead one to retrieve one of these incorrect associations, giving rise to a false recollection of having seen that actor perform that action earlier. Furthermore, the sameevent conjunction items should be falsely recognized with high confidence, given that recollection has been shown to be associated with high-confidence recognition responses (Yonelinas, 2001). Finally, Dodson, Bawa, and Slotnick (2007; see also Schacter, Koutstaal, Johnson, Gross, \& Angell, 1997) proposed that older adults are more prone to such false recollection than are young adults. In particular, they proposed that because of age-related hippocampal atrophy and a resulting disinhibition of binding processes, features of events occurring close together in time may become incorrectly bound, causing these incorrect pairings to be later recollected as if they had appeared together. It follows from this account that older adults may be especially prone to false recognition of the same-event conjunction items, because the two actors and their associated actions would be experienced in close temporal proximity, creating the possibility of incorrectly binding one actor with the actions of the other.

If, on the other hand, conjunction errors stem from the familiarity of an actor and action in the absence of recollection of the contexts in which they were encountered, as was suggested by the theory of Jones and Jacoby (2001), then participants should be equally likely to falsely recognize the same-event and different-event conjunction items. Both of these item types involved a familiar actor performing a familiar action, and thus they should elicit equivalent feelings of familiarity. Furthermore, if older adults' greater rate of false recognition of conjunction items stems from greater

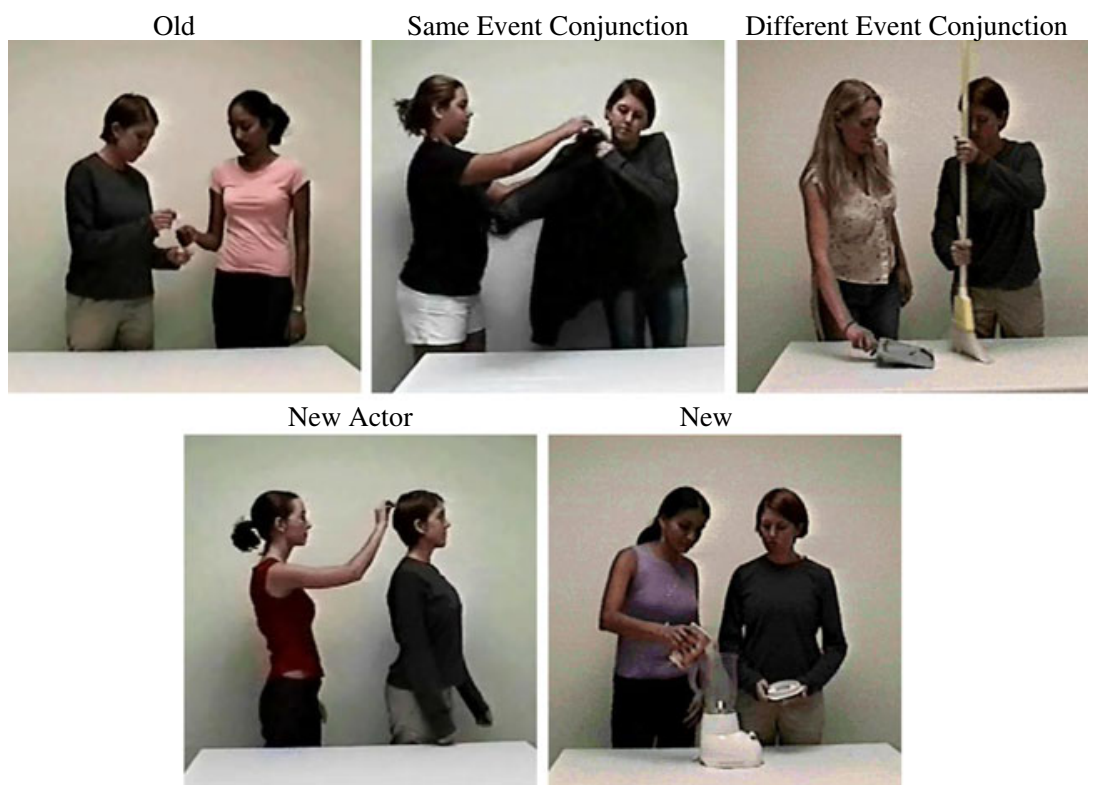

Fig. 2 Still frames from example test events 
reliance on familiarity in a recognition task, as was demonstrated by Jacoby (1999), then older adults should be more likely than young adults to falsely recognize both the sameevent and different-event conjunction items. Finally, if older adult recognition performance is driven primarily by familiarity rather than recollection, then increasing the familiarity levels of the actors and actions should increase older adults' rate of false recognition of novel conjunctions of those actors and actions.

To accomplish this last goal, we presented half of the events three times each at encoding and half of the events only once. Thus, half of the conjunction items that were presented later (the high-frequency items) involved an actor that had been seen performing the same action on three separate occasions, but now performing a different action that had been performed three separate times by a different actor. The other half of the conjunction items (the lowfrequency items) involved an actor seen only once performing an action that had previously been performed only once by a different actor. To the extent that their recognition performance is driven primarily by familiarity, older adults should be more likely to falsely recognize the high-frequency than the low-frequency conjunction items, because of the greater familiarity of the actors and actions appearing in the high-frequency items. Young adults, on the other hand, may be more likely to recollect the correct action that an actor had performed if that actor had performed that action on multiple occasions, thus allowing young adults to use recollection to reject a conjunction item involving that same actor now performing a different action. A higher frequency of presentation of the actors and actions appearing in a conjunction item may thus cause young adults to be less likely to falsely recognize that item, despite its greater familiarity, because young adults will be able to override this familiarity through recollection of the contexts in which that actor and action had appeared.

\section{Experiment 1}

Method

Participants A group of 32 young adults 18-22 years of age $(M=19.39)$ participated for course credit, and 32 highly educated, healthy older adults $60-87$ years of age $(M=$ 71.44) received $\$ 20$ gift certificates (see Table 1). An a priori power analysis was conducted using G*Power 3 (Faul, Erdfelder, Lang, \& Buchner, 2007) to determine the number of participants needed to achieve a statistical power of .95 to detect an interaction of age group and retrieval item type. Assuming an effect size of .2 and a correlation of .4 among repeated measures, this analysis revealed that 58 participants would be needed. In order to balance participants across the eight encoding lists, 64 participants were included in the experiment, resulting in an a priori power of .97 .

Stimuli Each of 265 video clips (mean duration $=6.07 \mathrm{~s}$ ) involved two female undergraduates taking part in a joint activity in which they played different roles. Eight encoding lists each involved 48 different videos portraying the actions of 96 different actors. Of the videos in each list, 24 were presented only once, whereas the other 24 were presented three times each. Eight retrieval lists each contained 60 videos. One of the actors in each retrieval item had not appeared in any of the encoding items. The same actor (hereafter termed Actor $X$ ) played this role in all 60 retrieval items. The purpose of employing Actor $X$ was to ensure that participants could only base their recognition judgments on the actions of one actor in each event. If the retrieval items had instead involved two actors who had both been seen at encoding, then switching the roles played by the two actors would have given participants two routes for rejecting the same-event conjunction items, with each action being performed by a different actor than the one who had

Table 1 Participant characteristics

\begin{tabular}{|c|c|c|c|c|c|c|c|}
\hline & \multicolumn{4}{|c|}{ Experiment 1} & \multirow[b]{3}{*}{$p$} & \multirow{2}{*}{\multicolumn{2}{|c|}{$\begin{array}{l}\text { Experiment } 2 \\
\text { Young Adults }\end{array}$}} \\
\hline & \multicolumn{2}{|c|}{ Older Adults } & \multicolumn{2}{|c|}{ Young Adults } & & & \\
\hline & Mean & $S D$ & Mean & $S D$ & & Mean & $S D$ \\
\hline Age & 71.44 & 6.51 & 19.39 & 0.95 & $<.001$ & 20.22 & 3.97 \\
\hline Education & 16.50 & 3.82 & 12.69 & 0.78 & $<.001$ & 12.55 & 1.37 \\
\hline Health & 4.03 & 0.90 & 4.48 & 0.62 & .02 & 4.17 & 0.75 \\
\hline Medications & 3.66 & 3.23 & 0.50 & 0.88 & $<.001$ & 0.30 & 0.76 \\
\hline Vocabulary & 36.40 & 2.20 & 27.91 & 3.21 & $<.001$ & 27.88 & 4.19 \\
\hline
\end{tabular}

Education $=$ number of years of education. Health $=$ self-reported health on a scale of 1 (poor) to 5 (excellent). Medications $=$ number of prescription medications currently being taken. Vocabulary $=$ score out of 40 on the Shipley (1986) Vocabulary test. $p=$ probability value associated with the comparison between young and older adults in Experiment 1 
performed that action at encoding. With Actor X instead playing one of the roles, participants' recognition judgments for all item types could only be based on the actions of the other actor in each event.

Five different types of retrieval items were presented (see Fig. 2). Twelve old items involved an actor performing the same action she had performed at encoding. Twelve sameevent conjunction items involved an actor appearing in the same event in which she had appeared at encoding, but now playing the opposite role. Twelve different-event conjunction items involved a familiar actor performing an action that had been performed by an actor in a different event at encoding. Twelve new-actor items involved a new actor performing a familiar action. Finally, 12 new items involved a new actor performing a new action.

Procedure The participants were instructed that they were to view a number of events on the computer screen and that they would later be tested on their memory for the events. They then viewed a total of 96 encoding items, with a different random order of presentation for each participant. After viewing each video clip, participants clicked on a button labeled "Next Event" to continue. After viewing the encoding events, participants were given a brief demographics questionnaire and a vocabulary test (Shipley, 1986).

After participants completed the vocabulary test, they were tested on their memory for the video clips seen earlier. The length of the retention interval separating the last encoding event from the first test event varied, depending on the speed with which participants completed the demographics questionnaire and vocabulary test. The retention interval for older adults (mean duration $=11.53 \mathrm{~min}, S D=$ $2.78 \mathrm{~min}$ ) was somewhat shorter than that for younger adults (mean duration $=13.94 \mathrm{~min}, S D=4.47 \mathrm{~min}$ ), $t(62)=2.59$, $p=.01$, reflecting the greater ease with which older adults completed the vocabulary test.

Before viewing the first test trial, participants were shown a picture of Actor X. They were instructed that Actor X had not appeared in the encoding events, and that they were to judge whether the other person appearing in each event was performing the same action that she had performed earlier. They were instructed that they would sometimes see an actor participating in the same event as before, but that she would now be playing the opposite role. Participants were instructed that they should only answer "yes" if the actor was playing the same role that she had played earlier. They were then presented with 60 retrieval items, with a different random order of presentation for each participant. After viewing each event, the participants were asked "Did you see this person play this role in the first part of the experiment?" After they had clicked "Yes" or "No," they were asked "How confident are you that you [saw (after responding positively)/did NOT see (after responding negatively)] this person play this role in the first part of the experiment?" and selected from among three buttons to indicate whether they were "just guessing," "pretty sure," or "absolutely sure."

\section{Results}

Figure 3 displays the proportions of "yes" responses and subsequent confidence ratings to the different item types by young and older adults. Table 2 presents these results broken down by presentation frequency. An alpha level of .05 was adopted for all analyses. An analysis of the proportions of
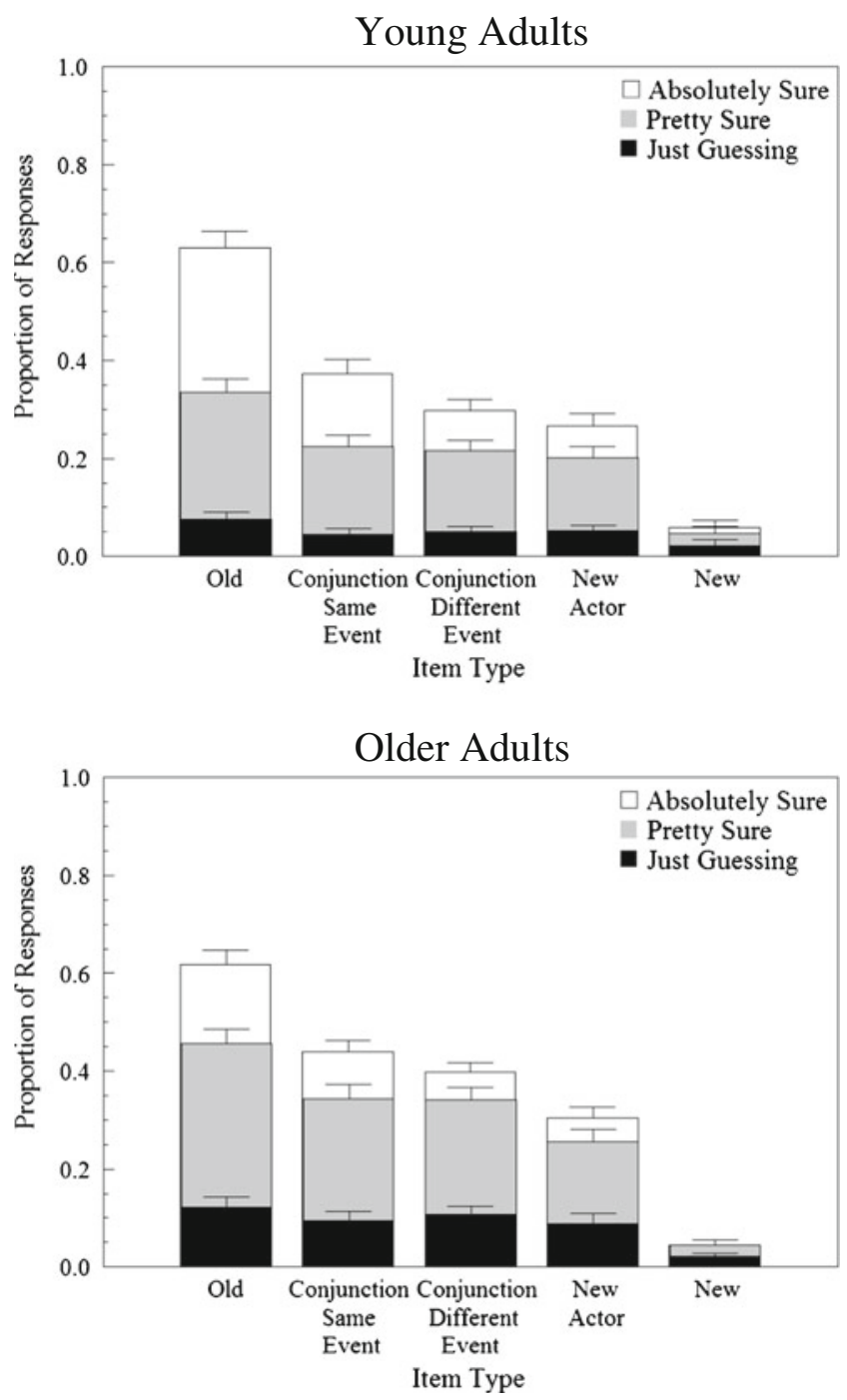

Fig. 3 Confidence in "yes" responses to the different item types. The height of each bar (summing across the three confidence categories) represents the overall proportion of "yes" responses to each item type. The composition of each bar represents the use of the different confidence ratings following those "yes" responses. Error bars represent the standard errors of the mean proportions of trials on which a given confidence rating was used 
Table 2 Proportions of "yes" responses and subsequent confidence ratings in Experiment 1

\begin{tabular}{|c|c|c|c|c|c|c|c|c|}
\hline \multirow[b]{3}{*}{ Item Type } & \multicolumn{4}{|c|}{ High-Frequency Items } & \multicolumn{4}{|c|}{ Low-Frequency and New Items } \\
\hline & \multicolumn{2}{|c|}{ Older Adults } & \multicolumn{2}{|c|}{ Young Adults } & \multicolumn{2}{|c|}{ Older Adults } & \multicolumn{2}{|c|}{ Young Adults } \\
\hline & Mean & $S D$ & Mean & $S D$ & Mean & $S D$ & Mean & $S D$ \\
\hline Old & 69 & .25 & .71 & .24 & .55 & .25 & .55 & .27 \\
\hline Absolutely sure & .22 & .21 & .41 & .27 & .10 & .18 & .18 & .17 \\
\hline Pretty sure or just guessing & .46 & .25 & .30 & .19 & .45 & .27 & .38 & .26 \\
\hline Same-event conjunction & .40 & .26 & .32 & .18 & .48 & .22 & .43 & .24 \\
\hline Absolutely sure & .12 & .21 & .15 & .20 & .07 & .13 & .15 & .20 \\
\hline Pretty sure or just guessing & .28 & .25 & .17 & .15 & .41 & .22 & .28 & .20 \\
\hline Different-event conjunction & .39 & .24 & .25 & .20 & .41 & .20 & .34 & .21 \\
\hline Absolutely sure & .06 & .13 & .08 & .13 & .05 & .14 & .08 & .18 \\
\hline Pretty sure or just guessing & .32 & .25 & .17 & .17 & .36 & .19 & .26 & .20 \\
\hline New actor & .28 & .27 & .20 & .24 & .33 & .22 & .33 & .22 \\
\hline Absolutely sure & .05 & .14 & .08 & .19 & .05 & .14 & .05 & .12 \\
\hline Pretty sure or just guessing & .23 & .26 & .13 & .14 & .28 & .21 & .28 & .20 \\
\hline New & & & & & .04 & .07 & .06 & .16 \\
\hline Absolutely sure & & & & & .00 & .00 & .01 & .07 \\
\hline Pretty sure or just guessing & & & & & .04 & .07 & .05 & .15 \\
\hline
\end{tabular}

High-frequency items involved actions seen on three separate occasions during encoding, with the same actor performing a given action throughout encoding. Low-frequency items involved actions seen only once. New items involved actors and actions not seen at encoding. The means and standard deviations printed in bold represent the overall proportions of "yes" responses to a given item type, which are subsequently broken down into "yes" responses followed by "absolutely sure" ratings of confidence, as well as "yes" responses followed by either "pretty sure" or "just guessing" ratings of confidence. Old items involved an actor performing the same action that she had performed previously. Same-event conjunction items involved an actor appearing in the same event in which she had appeared previously, but now playing the opposite role. Different-event conjunction items involved a familiar actor performing an action that had previously been performed by a different actor in a different event. New-actor items involved an unfamiliar actor performing an action that had been performed by a different actor. New items involved an unfamiliar actor performing an action that had not been seen at encoding

"yes" responses to new items revealed no significant difference between the two age groups, $t(62)=0.50, p>.10$, suggesting that the biases to respond "yes" were similar in the two groups. An analysis of variance (ANOVA) was then conducted on the proportions of "yes" responses to the remaining four item types, with item type, age group, and presentation frequency as independent variables.

The effects of item type were analyzed using three orthogonal planned comparisons (see Table 3). The first comparison contrasted performance on the new-actor items with performance on the old, same-event conjunction, and different-event conjunction items. This comparison measured a participant's ability to discriminate new from old actors. The second comparison contrasted performance on the old items with performance on the same-event conjunction and different-event conjunction items. This comparison measured a participant's ability to remember which actors performed which actions. Finally, the third comparison contrasted performance on the same-event conjunction items with performance on the different-event conjunction items. This comparison measured the association between an actor and characteristics of the event context in which she appeared.
Table 4 displays the results of applying these comparisons to the analysis of "yes" responses. When a significant effect was revealed for a given comparison, follow-up analyses involving the same comparison were conducted in which the total proportion of "yes" responses was separated into those followed by "absolutely sure" ratings of confidence and those followed by "pretty sure" or "just guessing" ratings of confidence. Recollection has been found to be associated with high confidence (Yonelinas, 2001), and thus if a participant were more likely to accept some item types than others on the basis of recollection, one would expect a comparison on these item types to remain significant even when analysis was limited to items that participants were "absolutely sure" they had seen before.

The analysis of "yes" responses revealed significant effects for all three of the planned comparisons. The first comparison revealed that participants were less likely to respond "yes" to the new-actor items than to the other three item types (contrast mean $=.17, S D=.17$ ), indicating that participants had some memory for the actors that they had seen. This comparison remained significant when the analysis was limited to "yes" responses followed by "absolutely sure" confidence ratings (contrast mean $=.08, S D=.09$ ), 
Table 3 Contrast weights for the orthogonal planned comparisons involving item type

\begin{tabular}{llll}
\hline & \multicolumn{2}{l}{ Comparison } & \\
\cline { 2 - 4 } Item Type & 1 & 2 & 3 \\
\hline Old & $1 / 3$ & 1 & 0 \\
Same-event conjunction & $1 / 3$ & $-1 / 2$ & 1 \\
Different-event conjunction & $1 / 3$ & $-1 / 2$ & -1 \\
New actor & -1 & 0 & 0 \\
\hline
\end{tabular}

Comparison 1 contrasted performance on the new-actor items with performance on the old, same-event conjunction, and different-event conjunction items. The new-actor items involved a new actor performing a familiar action, whereas the other item types involved familiar actors performing familiar actions. This comparison thus measured a participant's ability to discriminate old and new actors. Comparison 2 contrasted performance on the old items with performance on the same-event conjunction and different-event conjunction items. The old items involved an actor performing an action that she had performed earlier, whereas the other two item types involved an actor performing an action that had previously been performed by a different actor. This comparison thus measured a participant's ability to remember which actors had performed which actions. Comparison 3 contrasted performance on the same-event conjunction items with performance on the different-event conjunction items. In same-event conjunction items, an actor appeared in the same event context in which she had appeared earlier (albeit in the opposite role), whereas in different-event conjunction items, an actor appeared in a different event context. This comparison thus measured the association between an actor and the characteristics of the event context in which she appeared

suggesting that participants sometimes recollected having seen the actor in an old or conjunction item performing the same action earlier. This comparison also remained significant when analysis was limited to "yes" responses followed by intermediate confidence ratings (contrast mean $=.09$, $S D=.15$ ), suggesting that participants sometimes simply responded to the familiarity of the actors and actions in the old and conjunction items.

The second comparison revealed that participants were more likely to respond "yes" to the old items than to the two types of conjunction items (contrast mean $=.25, S D=.20$ ). This indicates that participants had some memory for which actors had performed which actions. This comparison remained significant when analysis was limited to "yes" responses followed by "absolutely sure" confidence ratings (contrast mean $=.13, S D=.18$ ), suggesting that participants were more likely to correctly recollect having seen the actor in an old item performing the same action previously than to falsely recollect having seen the actor in a conjunction item performing that same action previously. This comparison also remained significant when analysis was limited to "yes" responses followed by intermediate confidence ratings (contrast mean $=.11, S D=.15$ ). This suggests that participants may sometimes have been able to respond on the basis of the familiarity of a particular combination of actor and action, with old items involving familiar combinations and conjunction items involving unfamiliar combinations of actors and actions. This interpretation would be consistent with the notion of unitization of multiple features (in this case, an actor and an action) into a single, higher-level item, which could then potentially be recognized on the basis of familiarity (Diana, Yonelinas, \& Ranganath, 2008). This possibility will be explored further in the discussion.

The third comparison revealed that participants were more likely to falsely recognize the same-event than the differentevent conjunction items (contrast mean $=.06, S D=.18$ ), indicating that participants associated an actor with a particular event context, and thus were more likely to falsely recognize a novel pairing of a familiar actor and action if that actor and action had appeared together in the same event context. This comparison remained significant when analysis was limited to "yes" responses followed by "absolutely sure" confidence ratings (contrast mean $=.05, S D=.12$ ), but not when analysis was limited to "yes" responses followed by intermediate confidence ratings (contrast mean $=.01, S D=.17$ ). The significant comparison on "absolutely sure" responses suggests that participants were more likely to falsely recollect having seen an actor perform an action that had in fact been performed by somebody else if that actor and action had appeared together in the same event, consistent with the theory of Reinitz and Hannigan (2001).

Only the second comparison interacted with age. In particular, young adults (contrast mean $=.30, S D=.21$ ) performed better than older adults (contrast mean $=.20, S D=.17$ ) at discriminating old items from the two types of conjunction items, providing support for the general notion of an agerelated associative or binding deficit (Chalfonte \& Johnson, 1996; Naveh-Benjamin, 2000), and more specifically for an age-related deficit in the binding of actors with actions (Kersten et al., 2008; Kersten \& Earles, 2010). This interaction remained significant when analysis was limited to "yes" responses followed by "absolutely sure" confidence ratings, with young adults (contrast mean $=.18, S D=.19$ ) performing better than older adults (contrast mean $=.08, S D=.15$ ) at discriminating old and conjunction items in terms of "absolutely sure" responses. This result suggests that young adults were more likely than older adults to recollect having previously seen the old items and to discriminate them from conjunction items on that basis. This interaction was no longer significant when analysis was limited to "yes" responses followed by intermediate confidence ratings, with young adults (contrast mean $=.12, S D=.15$ ) performing similarly to older adults (contrast mean $=.11, S D=.15$ ) at discriminating old and conjunction items in terms of "pretty sure" and "just guessing" responses. The absence of this interaction appears to stem from an overall greater reliance on familiarity in older adults than in young adults, consistent with the theory of Jones and Jacoby (2001), with older adults more likely than 
Table 4 ANOVA results for Experiment 1

\begin{tabular}{|c|c|c|c|c|c|c|c|c|c|c|c|c|}
\hline \multirow[b]{2}{*}{ Effect } & \multicolumn{4}{|c|}{ All "Yes" Responses } & \multicolumn{4}{|c|}{ Absolutely Sure } & \multicolumn{4}{|c|}{ Pretty Sure or Just Guessing } \\
\hline & $F$ & $p$ & $M S E$ & $\eta_{\mathrm{p}}^{2}$ & $F$ & $p$ & $M S E$ & $\eta_{\mathrm{p}}^{2}$ & $F$ & $p$ & $M S E$ & $\eta_{\mathrm{p}}^{2}$ \\
\hline Age & 2.30 & $>.10$ & .033 & .04 & & & & & & & & \\
\hline Frequency & 1.25 & $>.10$ & .013 & .02 & & & & & & & & \\
\hline Age $\times$ Frequency & 0.98 & $>.10$ & .013 & .02 & & & & & & & & \\
\hline \multicolumn{13}{|l|}{ Item Type } \\
\hline Contrast 1 & 69.76 & $<.001$ & .056 & .53 & 57.72 & $<.001$ & .015 & .48 & 25.18 & $<.001$ & .043 & .29 \\
\hline Contrast 2 & 104.57 & $<.001$ & .075 & .63 & 37.53 & $<.001$ & .060 & .38 & 38.87 & $<.001$ & .043 & .39 \\
\hline Contrast 3 & 6.89 & .011 & .064 & .10 & 13.70 & $<.001$ & .027 & .18 & 0.02 & $>.10$ & .056 & .00 \\
\hline \multicolumn{13}{|l|}{ Age $\times$ Item Type } \\
\hline Contrast 1 & 0.09 & $>.10$ & .056 & .00 & & & & & & & & \\
\hline Contrast 2 & 4.10 & .047 & .075 & .06 & 4.85 & .031 & .060 & .07 & 0.01 & $>.10$ & .043 & .00 \\
\hline Contrast 3 & 0.58 & $>.10$ & .064 & .01 & & & & & & & & \\
\hline \multicolumn{13}{|c|}{ Frequency $\times$ Item Type } \\
\hline Contrast 1 & 5.15 & .027 & .044 & .08 & 9.32 & .003 & .012 & .13 & 0.49 & $>.10$ & .042 & .01 \\
\hline Contrast 2 & 23.13 & $<.001$ & .070 & .27 & 27.55 & $<.001$ & .032 & .31 & 1.92 & $>.10$ & .060 & .03 \\
\hline Contrast 3 & 0.37 & $>.10$ & .098 & .01 & & & & & & & & \\
\hline \multicolumn{13}{|c|}{ Age $\times$ Frequency $\times$ Item Type } \\
\hline Contrast 1 & 0.46 & $>.10$ & .044 & .01 & & & & & & & & \\
\hline Contrast 2 & 0.48 & $>.10$ & .070 & .01 & & & & & & & & \\
\hline Contrast 3 & 0.11 & $>.10$ & .098 & .00 & & & & & & & & \\
\hline
\end{tabular}

Significant effects in the analysis of "yes" responses are indicated in bold. In order to understand these significant effects, follow-up analyses were conducted in which "yes" responses were separated into those followed by "absolutely sure" ratings of confidence and those followed by "pretty sure" or "just guessing" ratings of confidence. Significant effects in these follow-up analyses are also indicated in bold. Contrast 1 on the item type variable compared new-actor items to old, same-event conjunction, and different-event conjunction items, measuring memory for actors. Contrast 2 compared old items to same-event conjunction and different-event conjunction items, measuring memory for which actors were associated with which actions. Contrast 3 compared same-event conjunction and different-event conjunction items, measuring the association between an actor and the event context in which she appeared

young adults to respond "pretty sure yes" or "just guessing yes," both to the old items, $t(62)=2.62, p=.01$, and to the conjunction items, $t(62)=3.68, p<.001$.

Significant interactions with presentation frequency were revealed for the first and second planned comparisons involving item type, both of which involved a contrast between old items and one or more other item types. Most notably, with regard to the second planned comparison, participants were more likely to correctly accept old items that had been seen on multiple occasions rather than only once, $t(63)=3.68, p<.001$, whereas they were less likely to falsely recognize the conjunction items when they involved actors and actions seen on multiple occasions rather than only once, $t(63)=2.97, p=.004$. This interaction remained significant when the analysis was limited to "yes" responses followed by "absolutely sure" confidence ratings, but not when the analysis was limited to "yes" responses followed by intermediate confidence ratings. These results suggest that participants were more likely to recollect having seen an actor perform a particular action when that actor had performed that action on multiple occasions. This would lead participants to correctly accept the old items with high confidence, whereas it would lead participants to correctly reject the conjunction items, because they would remember having seen a different actor perform that action at encoding.

Finally, we observed no three-way interactions involving presentation frequency, item type, and age group, suggesting that increases in presentation frequency led to greater discrimination of old items from the other item types in both young and older adults. Seeing the same actor perform the same action on multiple occasions thus sometimes allowed older adults as well as young adults to later recollect having seen that actor perform that action, allowing them to reject an event involving that actor performing a different action.

\section{Discussion}

The results of Experiment 1 provide evidence that both of the mechanisms that have been proposed to explain conjunction memory errors may sometimes lead to errors in memory for who did what in an event. In particular, 
consistent with the theory of Reinitz and Hannigan (2001, 2004), participants were more likely to falsely recognize an actor performing an action that had previously been performed by somebody else within the same event than by somebody from a different event. This suggests that the simultaneous presence of an actor and another person's actions caused participants to sometimes incorrectly associate that actor with the other person's actions. Moreover, the higher rate of false recognition of the same-event than of the different-event conjunction items was exclusively associated with "absolutely sure" confidence ratings, suggesting that the incorrect association between an actor and another person's actions led participants to later falsely recollect having seen that actor perform those actions.

The pattern of age differences observed in this experiment, however, also provides evidence for the theory of Jones and Jacoby (2001). In particular, older adults were more likely than young adults to falsely recognize both types of conjunction items, with this higher rate of false recognition associated with intermediate levels of confidence. Older adults were also more likely than young adults to accept the old items at these intermediate levels of confidence. This pattern of results suggests that the combination of a familiar actor and a familiar action, regardless of whether or not they had gone together at encoding, caused older adults to experience a feeling of familiarity, leading them to believe that they had previously seen that actor perform that action. Young adults presumably experienced this same feeling of familiarity, but were more likely to recollect the true sources of this familiarity (e.g., the actor who had in fact performed the action), thus allowing them to accept the old items with higher confidence and to reject the conjunction items despite the familiarity that they engendered.

Although the results of Experiment 1 provided evidence for a false recollection process as well as for an influence of familiarity, the evidence for false recollection was somewhat indirect. Yonelinas (2001) proposed that recollection is associated with high confidence, and thus the finding that participants' higher rates of false recognition of the sameevent than of the different-event conjunction items were associated with "absolutely sure" confidence ratings suggests that participants at least sometimes falsely recollected having seen the actor in a same-event conjunction item performing that same action earlier. Familiarity may also sometimes lead to high-confidence recognition judgments, however. In particular, the familiarity levels associated with a set of recognition items are thought to be distributed normally around the mean level of familiarity for that type of item (Yonelinas, 2001). Thus, if the familiarity level of a given item falls on the extreme positive tail of this distribution, it may elicit a high-confidence recognition judgment even in the absence of recollection.
One possible familiarity-based account of the difference in the rates of false recognition of the same-event and different-event conjunction items is that participants may have been able to form a unitized representation of an actor and the event context in which she appeared. Diana et al. (2008; see also Hayes, Nadel, \& Ryan, 2007) argued that it is possible under certain encoding conditions to unitize, or fuse together, item information with contextual information. These unitized representations can then support recognition on the basis of familiarity, with the information in a test item either matching or failing to fully match the information in the fused representation of item and context.

Evidence from the present experiment for such a unitization process would appear to come from the contrast between old items and the two types of conjunction items, with both young and older adults being more likely to make "pretty sure yes" and "just guessing yes" responses to old than to conjunction items. This result suggests that participants were sometimes able to unitize an actor with the action that she performed. Thus, when this actor was later seen performing that same action (in an old item), participants may have experienced a feeling of familiarity. In contrast, when this actor was seen performing a different action (in a conjunction item), they may have been less likely to experience this feeling of familiarity, leading to a difference in the rate of acceptance of these two item types, with this difference being localized at intermediate levels of confidence.

Given this suggestion of a unitization process operating on the present stimuli, it remains possible that unitization could also explain the difference in the rates of false recognition of the two types of conjunction items. In particular, given the simultaneous presence of an actor and an action performed by another person, participants may have fused these two pieces of information into a single, unitized representation, leading them to later falsely recognize, on the basis of familiarity, a test event involving those same two pieces of information (i.e., a same-event conjunction item). In contrast, participants may have been less likely to unitize an actor with the actions of another person appearing in a different event, causing them to be less likely to falsely recognize a test event involving those two pieces of information (i.e., a different-event conjunction item). It is unclear by this account why the difference between the same-event and different-event conjunction items would be most evident at the highest level of confidence, but it is possible that the match between a same-event conjunction item and a unitized representation of an actor and an action may have been strong enough to yield a familiarity signal that fell on the extreme positive tail of the familiarity distribution, leading to a high-confidence "yes" response.

Further evidence was thus needed to determine whether the higher acceptance of same-event than of different-event conjunction items is truly associated with false recollection, 
or whether it simply reflects high levels of familiarity for the same-event conjunction items stemming from a match of these items with unitized representations of previously encountered actors and actions. Experiment 2 was designed to address this need.

\section{Experiment 2}

Experiment 2 applied a variant of Tulving's (1985) remember-know procedure to the stimuli of Experiment 1. This allowed us to test whether participants' greater rate of acceptance of the same-event than of the different-event conjunction items was accompanied by a phenomenological experience of "remembering" a prior event (e.g., bringing to mind a visual image of the actor performing the action), as opposed to simply "knowing" that the event had been encountered before (i.e., receiving a feeling of familiarity for it) without specifically recollecting it. If the greater rate of acceptance of the same-event than of the different-event conjunction items reflects false recollection of having seen that same actor perform that same action previously, as is suggested by the theory of Reinitz and Hannigan (2001, 2004), participants should make more "remember" responses to the same-event than to the different-event conjunction items. In contrast, if the greater rate of acceptance of the same-event than of the different-event conjunction items reflects high levels of familiarity for the same-event conjunction items, participants should make more "know" responses to the same-event than to the different-event conjunction items.

The primary goal of Experiment 2 was thus to explain the difference in the rates of false recognition of the same-event and different-event conjunction items. Young and older adults did not differ significantly in the extents to which they differentiated these two item types in Experiment 1. Thus, only young adults were tested in Experiment 2.

\section{Method}

Participants A group of 152 undergraduates 18-48 years of age (mean $=20.22$ years) participated for course credit (see Table 1). A priori power analysis was conducted to determine the number of participants needed to achieve statistical power of .95 to reveal a significant difference between the same-event and different-event conjunction items with a two-tailed test. The effect size for this comparison was estimated at 0.3 , on the basis of results from Experiment 1. This analysis revealed that 147 participants would be needed. In order to balance participants across eight encoding lists, 152 participants were included in the experiment, resulting in a priori power of .96 .
Stimuli and procedure The encoding and retrieval lists were identical to those of Experiment 1. The test format for the retrieval phase of the experiment differed from that of Experiment 1, however. In particular, when participants responded "yes" to the question "Did you see this person play this role in the first part of the experiment?" they were subsequently asked to make a judgment about the phenomenological experience of memory retrieval that led them to a positive recognition judgment, adapted from Tulving's (1985) remember-know paradigm. In particular, participants were asked whether they recollected having seen the actor perform that same action earlier, or whether they simply received a feeling of familiarity from viewing the actor perform that action (see the Appendix for detailed instructions).

\section{Results}

Figure 4 displays the proportions of "yes" responses to the different item types and subsequent attributions of those responses to recollection and familiarity. Table 5 presents these results broken down by presentation frequency. An ANOVA was conducted on the proportions of "yes" responses to the old, same-event conjunction, different-event conjunction, and new-actor items, with item type and presentation frequency as independent variables. The effects of item type were analyzed with the same three orthogonal planned comparisons that had been used in Experiment 1.

Table 6 displays the results of applying these comparisons to the analysis of "yes" responses. When a significant effect

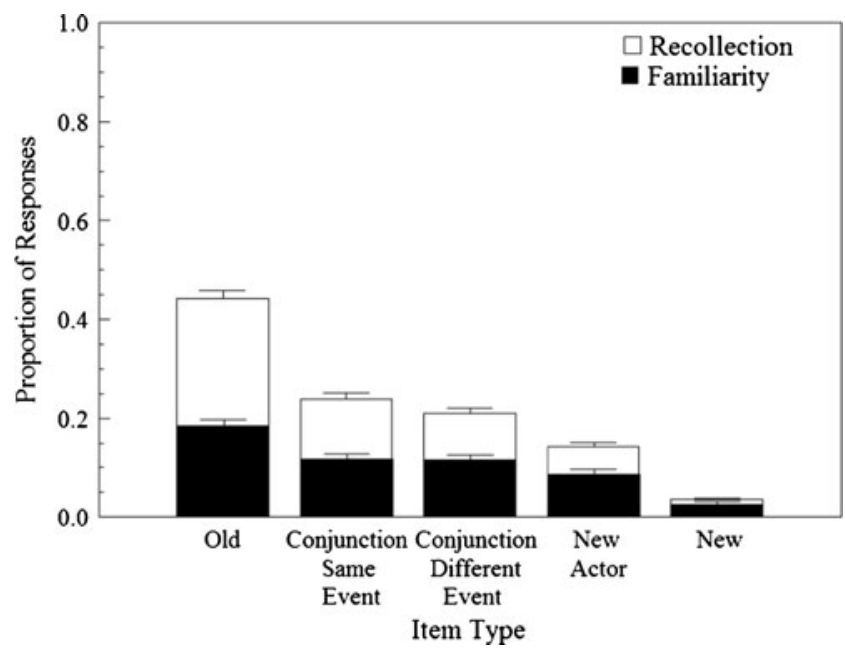

Fig. 4 Proportions of trials on which "yes" responses to the different item types were attributed to familiarity and recollection. The height of each bar (summing across familiarity and recollection) represents the overall proportion of "yes" responses to each item type. The composition of each bar represents the use of familiarity and recollection to justify those "yes" responses. Error bars represent the standard errors of the mean proportions of trials on which a given justification was used 
Table 5 Proportions of "yes" responses and subsequent rememberknow judgments in Experiment 2

\begin{tabular}{|c|c|c|c|c|}
\hline \multirow[b]{2}{*}{ Item Type } & \multicolumn{2}{|c|}{$\begin{array}{l}\text { High-Frequency } \\
\text { Items }\end{array}$} & \multicolumn{2}{|c|}{$\begin{array}{l}\text { Low-Frequency } \\
\text { and New Items }\end{array}$} \\
\hline & Mean & $S D$ & Mean & $S D$ \\
\hline Old & .49 & .33 & .40 & .25 \\
\hline Remember & .32 & .28 & .19 & .20 \\
\hline Know & .16 & .20 & .20 & .19 \\
\hline Same-event conjunction & .21 & .25 & .27 & .23 \\
\hline Remember & .13 & .19 & .12 & .16 \\
\hline Know & .09 & .14 & .15 & .18 \\
\hline Different-event conjunction & .19 & .23 & .23 & .22 \\
\hline Remember & .10 & .17 & .09 & .14 \\
\hline Know & .09 & .15 & .14 & .18 \\
\hline New actor & .11 & .17 & .17 & .20 \\
\hline Remember & .04 & .10 & .07 & .14 \\
\hline Know & .07 & .14 & .10 & .15 \\
\hline New & & & .04 & .08 \\
\hline Remember & & & .01 & .03 \\
\hline Know & & & .03 & .07 \\
\hline
\end{tabular}

High-frequency items involved actions seen on three separate occasions during encoding, with the same actor performing a given action throughout encoding. Low-frequency items involved actions seen only once. New items involved actors and actions not seen at encoding. The means and standard deviations printed in bold represent the overall proportions of "yes" responses to a given item type, which are subsequently broken down into "yes" responses followed by "remember" judgments, as well as "yes" responses followed by "know" judgments. Old items involved an actor performing the same action that she had performed previously. Same-event conjunction items involved an actor appearing in the same event in which she had appeared previously, but now playing the opposite role. Different-event conjunction items involved a familiar actor performing an action that had previously been performed by a different actor in a different event. New-actor items involved an unfamiliar actor performing an action that had been performed by a different actor. New items involved an unfamiliar actor performing an action that had not been seen at encoding

was revealed for a given comparison, follow-up analyses involving the same comparison were conducted in which the total proportion of "yes" responses was separated into those followed by "remember" judgments and those followed by "know" judgments. The analysis of "yes" responses again revealed significant effects for all three of the planned comparisons. The first comparison revealed that participants were less likely to respond "yes" to the new-actor items than to the other three item types (contrast mean $=.15, S D=.14$ ). This comparison remained significant when analysis was limited to "yes" responses followed by "remember" judgments (contrast mean $=.10, S D=.10$ ), suggesting that participants sometimes recollected having seen the actor in an old or conjunction item performing the same action earlier. This comparison also remained significant when analysis was limited to "yes" responses followed by "know" judgments (contrast mean = $.05, S D=.10$ ), suggesting that participants sometimes simply responded to the familiarity of the actors and actions in the old and conjunction items.

The second comparison revealed that participants were more likely to respond "yes" to the old items than to the two types of conjunction items (contrast mean $=.22, S D=.22$ ). This comparison remained significant when analysis was limited to "yes" responses followed by "remember" judgments (contrast mean $=.15, S D=.18$ ). This suggests that participants were more likely to correctly recollect having seen the actor in an old item performing the same action previously than to falsely recollect having seen the actor in a conjunction item performing that same action previously. This comparison also remained significant when analysis was limited to "yes" responses followed by "know" judgments (contrast mean $=.07, S D=.14$ ). This suggests that participants may sometimes have been able to respond on the basis of the familiarity of a particular combination of actor and action, with old items involving familiar combinations and conjunction items involving unfamiliar combinations of actors and actions. This finding thus provides further support for the notion of unitization of actor and action information.

Finally, the third comparison revealed that participants were more likely to falsely recognize the same-event conjunction items than to falsely recognize the different-event conjunction items (contrast mean $=.03, S D=.18$ ). This comparison remained significant when analysis was limited to "yes" responses followed by "remember" judgments (contrast mean $=.03, S D=.15$ ), but not when analysis was limited to "yes" responses followed by "know" judgments (contrast mean $=.00, S D=.13$ ). The significant contrast on "remember" judgments suggests that participants were more likely to falsely recollect having seen an actor perform an action that had previously been performed by somebody else if that actor and action had appeared together in the same event, consistent with the theory of Reinitz and Hannigan (2001). The absence of a difference in the use of "know" judgments with the two types of items, on the other hand, provides no evidence for unitization of actor information with information about the actions of another person performed within the same context, which might have allowed participants to distinguish the two types of items on the basis of familiarity.

Significant interactions with presentation frequency were revealed for both the first and the second planned comparisons involving item type, both of which involved a contrast between old items and one or more other item types. Furthermore, both of these interactions remained significant when the analysis was limited to "yes" responses followed by "remember" judgments, suggesting that participants were more likely to recollect having seen an actor perform a 
Table 6 ANOVA results for Experiment 2

\begin{tabular}{|c|c|c|c|c|c|c|c|c|c|c|c|c|}
\hline \multirow[b]{2}{*}{ Effect } & \multicolumn{4}{|c|}{ All "Yes" Responses } & \multicolumn{4}{|c|}{ "Remember" Judgments } & \multicolumn{4}{|c|}{ "Know" Judgments } \\
\hline & $F$ & $p$ & $M S E$ & $\eta_{\mathrm{p}}^{2}$ & $F$ & $p$ & $M S E$ & $\eta_{\mathrm{p}}^{2}$ & $F$ & $p$ & $M S E$ & $\eta_{\mathrm{p}}^{2}$ \\
\hline Frequency & 2.02 & $>.10$ & .008 & .01 & & & & & & & & \\
\hline \multicolumn{13}{|l|}{ Item Type } \\
\hline Contrast 1 & 185.48 & $<.001$ & .039 & .55 & 144.98 & $<.001$ & .022 & .49 & 39.27 & $<.001$ & .021 & .21 \\
\hline Contrast 2 & 143.08 & $<.001$ & .100 & .49 & 100.91 & $<.001$ & .067 & .40 & 34.71 & $<.001$ & .040 & .19 \\
\hline Contrast 3 & 4.15 & .043 & .064 & .03 & 4.89 & .028 & .047 & .03 & 0.04 & $>.10$ & .034 & .00 \\
\hline \multicolumn{13}{|c|}{ Frequency $\times$ Item Type } \\
\hline Contrast 1 & 11.95 & .001 & .025 & .07 & 43.27 & $<.001$ & .013 & .22 & 2.37 & $>.10$ & .018 & .02 \\
\hline Contrast 2 & 28.06 & $<.001$ & .050 & .16 & 34.67 & $<.001$ & .031 & .19 & 0.85 & $>.10$ & .027 & .01 \\
\hline Contrast 3 & 0.47 & $>.10$ & .063 & .00 & & & & & & & & \\
\hline
\end{tabular}

Significant effects in the analysis of "yes" responses are indicated in bold. In order to understand these significant effects, follow-up analyses were conducted in which "yes" responses were separated into those followed by "remember" judgments and those followed by "know" judgments. Significant effects in these follow-up analyses are also indicated in bold. Identical patterns of significant effects were obtained when analyzing the raw numbers of "remember" and "know" judgments and when analyzing estimates of recollection and familiarity derived from these numbers using Yonelinas and Jacoby's (1995) independent remember-know method. Thus, for simplicity, only the analysis of raw scores is reported. Contrast 1 on the item type variable compared new-actor items to old, same-event conjunction, and different-event conjunction items, measuring memory for actors. Contrast 2 compared old items to same-event conjunction and different-event conjunction items, measuring memory for which actors were associated with which actions. Contrast 3 compared same-event conjunction and different-event conjunction items, measuring the association between an actor and the event context in which she appeared

particular action when that actor had performed that action on multiple occasions.

\section{Discussion}

Experiment 2 replicated the finding from Experiment 1 that participants were more likely to falsely recognize an actor performing an action that had previously been performed by somebody else if those two actors had appeared together in the same event. Participants were more conservative overall in Experiment 2 than in Experiment 1, perhaps because they were not allowed to indicate that they were "just guessing" when they made a "yes" responses, and thus may have responded "no" when they could not justify a "yes" response on the basis of either familiarity or recollection. Despite these overall lower rates of both correct and incorrect acceptances of the recognition items, all of the same patterns emerged in the comparison of the different item types. Moreover, Experiment 2 demonstrated that the greater rate of false recognition of the same-event than of the different-event conjunction items was associated with the phenomenological experience of "remembering," or specifically recollecting having seen an actor perform the same action previously. These results are consistent with the theory of Reinitz and Hannigan (2001, 2004) that the simultaneous presence of two stimuli-in this case, of an actor and the actions of another person-can cause one to later falsely recollect having seen those two features together as parts of the same stimulus.

\section{General discussion}

\section{Conjunction memory errors}

The present results provide evidence for two independent influences on the likelihood of incorrectly attributing an action to an actor who had in fact been seen doing something else. One influence may involve feelings of familiarity for an actor and an action in the absence of recollection of the contexts in which that actor and action were encountered, consistent with the theory of conjunction errors proposed by Jones and Jacoby (2001). Evidence for this influence came from the finding that older adults were more likely than young adults to falsely recognize both types of conjunction items, with this higher rate of false recognition being associated with "pretty sure" and "just guessing" responses. Because the use of intermediate points on a rating scale has previously been found to be associated with familiarity rather than recollection (Yonelinas, 2001), this result suggests that older adults' increased rate of false recognition of conjunction items stemmed from the familiarity of the features of those items. Both young and older adults were sometimes able to override this feeling of familiarity for the conjunction items with recollection of the actual action that an actor had performed (especially if that actor had been seen performing that action on multiple occasions), allowing them to reject an item involving that actor now performing a different action. Young adults were evidently more proficient than older adults at using this 
recollection-to-reject process, however, leaving older adults more reliant on the familiarity of the individual features of these items when judging whether or not they had seen the items before.

A second influence on the likelihood of falsely recognizing conjunction items may involve false recollection of having seen an actor perform an action that had in fact been performed by somebody else, consistent with the theory of conjunction errors proposed by Reinitz and Hannigan (2001). Evidence for this influence came from the greater likelihood of falsely associating an actor with the actions of somebody else when that actor had in fact been present in a different role when those actions were carried out. This increased likelihood of falsely associating an actor with the actions of another person within the same event was associated with "absolutely sure" responses in Experiment 1 and with "remember" responses in Experiment 2. These results suggest that the combination of a familiar actor and the actions of another person from within that same event sometimes caused participants to recollect having seen that actor perform those actions.

A possible mechanism underlying this finding is that the actor and the action appearing in a same-event conjunction item provided two different retrieval cues for the original event that was seen at encoding, making participants particularly likely to recollect this prior event. Recollection can never provide a perfectly detailed reconstruction of an event, however, with attentional limitations preventing some information in the event from being encoded. In the present research, participants may have correctly encoded the appearances of the two actors in an event and the two roles that were played, but may have failed to correctly encode the correspondences between the two actors and the two roles. As a result, when participants later recollected the event, the roles played by the two actors may have been reversed. This account thus suggests that false recognition of the same-event conjunction items in the present research may be quite similar to illusory conjunctions in perception (Treisman \& Schmidt, 1982), in which attentional limitations lead to features from two different objects being incorrectly bound together.

This notion of partial recollection may also explain why participants sometimes falsely recognized the differentevent conjunction and new-actor items with high confidence in Experiment 1, and sometimes made "remember" judgments to these items in Experiment 2. In particular, the familiar action in these items may have served as a retrieval cue for the original event in which this same action was performed, but participants may have failed to encode the appearances of the actors who had participated in the earlier event. This retrieval of action information, in the absence of conflicting information about the identities of the actors who had carried out that action, may have led participants to a phenomenological experience of remembering, causing them to strongly believe that they had encountered this event before. Thus, although the same-event conjunction items may have been particularly likely to evoke this experience of remembering because of the presence of multiple retrieval routes to the original event, the presence of a familiar action in the different-event conjunction and newactor items may also sometimes have been sufficient to elicit false recollection.

Experiment 1 provides no evidence for age differences in the likelihood of false recollection of conjunction items, with no significant differences between the two age groups in the use of "absolutely sure yes" responses to the conjunction items. The present results thus provide no support for the theory that older adults are more prone to false recollection than are young adults (Dodson et al., 2007), at least in the context of the present stimuli. In fact, we observed a trend toward greater use of "absolutely sure yes" responses to the conjunction items by young than by older adults. One could perhaps argue that an increase in false recollection in older adults was offset by other factors (e.g., anxiety about memory decline; see Earles \& Kersten, 1998; Earles, Kersten, Más, \& Miccio, 2004) that served to decrease their overall confidence in their memory abilities, leading them to assign lower confidence ratings to their recognition responses, even when those responses were based on recollection. The most straightforward explanation for the present results, however, is that older adults' recollection deficit primarily involves a failure to correctly recollect target items rather than a tendency to falsely recollect lure items (a conclusion also recently reached by Wong, Cramer, \& Gallo, 2012).

\section{Implications for eyewitness testimony}

The present research suggests that eyewitnesses may sometimes misremember the roles played by different actors within an event. This could have important implications for eyewitness testimony, in which memory for who did what is crucial. The few previous studies that have examined eyewitness memory for roles within an event (e.g., Geiselman, Haghighi, \& Stown, 1996; Read et al., 1990; Wells \& Pozzulo, 2006), however, have found little evidence for such "role transference," in contrast to the present study.

However, a number of procedural differences between the present research and these prior studies could account for the different results that were obtained; these procedural differences included the use of an event recognition as opposed to a lineup identification task, the use of a larger number of actors, and testing memory for a larger number of events. Perhaps most notable among these differences, however, was that the earlier studies involved actions that were either clearly criminal in nature (e.g., purse snatchings in the 
Geiselman et al., 1996, and Wells \& Pozzulo, 2006, studies) or at least quite unusual (e.g., asking for \$20 in quarters in the Read et al., 1990, study), whereas the actions in the present research were mundane and ordinary. It is possible that the uniqueness of the actions in the earlier studies caused participants to have little difficulty distinguishing the perpetrators of these actions from the perpetrators of other, more typical actions.

One may argue that the uniqueness of the actions employed in the earlier studies may have made them more relevant to real-world eyewitness testimony, in which an eyewitness may be exposed to violent, disturbing actions that he or she will hopefully not experience at any other time. A great deal of eyewitness testimony, however, centers on actions that may not have been perceived to be especially unusual at the time (e.g., who was seen where at what time). Thus, even if the present research is not directly applicable to memory for emotionally laden violent crimes, the present findings are quite relevant to the arguably much more common situation in which an eyewitness is asked for testimony regarding everyday types of actions that he or she observed.

\section{Conclusions}

The present results provide evidence that both young and older adults will sometimes incorrectly recollect the role played by an actor within an event, falsely associating that actor with the actions of another person within the same event. They also provide evidence that, on the basis of familiarity, older adults in particular, but sometimes young adults as well, will falsely attribute an action to an actor who had been seen doing something else. This combination of findings suggests that an innocent bystander, seen in an everyday context, or possibly at a crime scene, may sometimes be remembered as having performed an action that had actually been performed by somebody else, with both mechanisms potentially leading a witness to believe that the bystander had performed those actions.

Author note This research was supported in part by grants from the National Institute on Aging and from Florida Atlantic University.

\section{Appendix: Test instructions for Experiment 2}

You will now be tested on your memory for the events you saw earlier. To do this, we will present you with a number of new events. One of the people in each of these new events will be the person appearing to the right. You did not see this person in the first part of the experiment, and so you are not being tested on your memory for this person. We are instead interested in your memory for the other person appearing in each event. In particular, we would like to know if you remember having seen this other person in the first part of the experiment, and if so, if that person is doing the same thing she was seen doing in the first part of the experiment.

This memory test will be difficult, because sometimes you will see a person taking part in the same event in which she took part earlier in the experiment, but she will be playing the opposite role. For example, if you saw one person tickling a second person in the first part of the experiment, you might now be presented with an event in which the second person is now doing the tickling (rather than being tickled). After seeing this event, you will be asked "Did you see this person play this role in the first part of the experiment?" In this example, you would answer "No" to this question, because the person in question is now playing the opposite role (i.e., tickling rather than being tickled).

If you click the "Yes" button to indicate that you did indeed see the person in the video clip playing the same role earlier in the experiment, you will then be asked on what basis you made your decision. In particular, could you consciously recollect having seen that person play that same role earlier? Alternatively, does the sight of this person playing this role evoke such a strong feeling of familiarity that you believe you must have seen this person play this role earlier, even though you can't consciously recollect having seen this person play this role? You will be asked to choose which of these two reasons better explains your decision that you did indeed see this person play this role earlier.

The strongest basis on which to make your decision that you saw a person play the same role earlier is that you can consciously recollect having seen that same person performing those same actions earlier. To recollect is to become consciously aware again of what happened or what was experienced at the time you first saw the person (e.g., what the person looked like when you first saw her, what sorts of facial expressions or body movements she exhibited, or what you were thinking about when you first saw her perform these actions). In other words, a recollected event should bring back to mind a particular association, mental image, or something more personal from the time of study, or something about the timing or position of the event within the study list.

Another possible basis on which to make your decision that you saw a person play the same role earlier is that the sight of that person playing that role at test evokes a strong feeling of familiarity. This feeling of familiarity may cause you to believe that you must have seen that person play that role earlier, even though you cannot consciously recollect having seen that person play that role. In other words, you should indicate that an event is familiar when you are confident that you saw the actor in an event play the same role earlier, but this event fails to evoke any specific conscious recollection from the time of study. 
To further clarify the difference between these different bases for deciding that you saw a person play the same role earlier, here are some examples. If a friend asked you what happened in a movie you saw last night, you would probably recollect the actions of the different characters, because you would be able to bring to mind visual images of the characters performing those actions. If, on the other hand, you saw someone standing outside of class who was really familiar but you had no recollection of seeing that person before (i.e., déjà vu), you might decide on the basis of familiarity that you must have seen that person standing outside of class before.

Do you understand the difference between recognizing a person's actions on the basis of conscious recollection or on the basis of a feeling of familiarity? If not, please ask the experimenter for further instructions. Otherwise, please click on the right arrow key to begin the test.

\section{References}

Buckhout, R. (1974). Eyewitness testimony. Scientific American, 231, 23-31.

Chalfonte, B. L., \& Johnson, M. K. (1996). Feature memory and binding in young and older adults. Memory \& Cognition, 24, 403-416. doi:10.3758/BF03200930

Davis, D., Loftus, E. F., Vanous, S., \& Cucciare, M. (2008). Unconscious transference" can be an instance of "change blindness. Applied Cognitive Psychology, 22, 605-623. doi:10.1002/ acp. 1395

Diana, R. A., Yonelinas, A. P., \& Ranganath, C. (2008). The effects of unitization on familiarity-based source memory: Testing a behavioral prediction derived from neuroimaging data. Journal of Experimental Psychology: Learning, Memory, and Cognition, 34, 730-740. doi:10.1037/0278-7393.34.4.730

Dodson, C. S., Bawa, S., \& Slotnick, S. D. (2007). Aging, source memory, and misrecollections. Journal of Experimental Psychology: Learning, Memory, and Cognition, 33, 169-181. doi:10.1037/0278-7393.33.1.169

Earles, J. L., \& Kersten, A. W. (1998). Influences of age and perceived activity difficulty on activity recall. Journals of Gerontology, 53B, P324-P328. doi:10.1093/geronb/53B.5. P324

Earles, J. L., Kersten, A. W., Curtayne, E. S., \& Perle, J. G. (2008). That's the man who did it, or was it a woman? Actor similarity and binding errors in event memory. Psychonomic Bulletin \& Review, 15, 1185-1189. doi:10.3758/PBR.15.6.1185

Earles, J. L., Kersten, A. W., Más, B. B., \& Miccio, D. M. (2004). Aging and memory for self-performed tasks: Effects of task difficulty and time pressure. Journals of Gerontology, 59, P285P293. doi:10.1093/geronb/59.6.P285

Faul, F., Erdfelder, E., Lang, A., \& Buchner, A. (2007). G*Power 3: A flexible statistical power analysis program for the social, behavioral, and biomedical sciences. Behavior Research Methods, 39, 175-191. doi:10.3758/BF03193146
Geiselman, R. E., Haghighi, D., \& Stown, R. (1996). Unconscious transference and characteristics of accurate and inaccurate eyewitnesses. Psychology, Crime \& Law, 2, 197-209.

Hayes, S. M., Nadel, L., \& Ryan, L. (2007). The effect of scene context on episodic object recognition: Parahippocampal cortex mediates memory encoding and retrieval success. Hippocampus, 17, 873-889.

Jacoby, L. L. (1999). Ironic effects of repetition: Measuring age-related differences in memory. Journal of Experimental Psychology: Learning, Memory, and Cognition, 25, 3-22. doi:10.1037/02787393.25.1.3

Jones, T. C., \& Jacoby, L. L. (2001). Feature and conjunction errors in recognition memory: Evidence for dual-process theory. Journal of Memory and Language, 45, 82-102. doi:10.1006/jmla.2000.2761

Kersten, A. W., \& Earles, J. L. (2010). Effects of aging, distraction, and response pressure on the binding of actors and actions. Psychology and Aging, 25, 620-630. doi:10.1037/a0019131

Kersten, A. W., Earles, J. L., Curtayne, E. S., \& Lane, J. C. (2008). Adult age differences in binding actors and actions in memory for events. Memory \& Cognition, 36, 119-131. doi:10.3758/MC.1.119

Loftus, E. F. (1976). Unconscious transference in eyewitness identification. Law and Psychology Review, 2, 93-98.

Naveh-Benjamin, M. (2000). Adult age differences in memory performance: Tests of an associative deficit hypothesis. Journal of Experimental Psychology: Learning, Memory, and Cognition, 26, 1170-1187. doi:10.1037/0278-7393.26.5.1170

Read, J. D., Tollestrup, P., Hammersley, R., McFadzen, E., \& Christensen, A. (1990). The unconscious transference effect: Are innocent bystanders ever misidentified? Applied Cognitive Psychology, 4, 3-31.

Reinitz, M. T., \& Hannigan, S. L. (2001). Effects of simultaneous stimulus presentation and attention switching on memory conjunction errors. Journal of Memory and Language, 44, 206-219. doi:10.1006/jmla.2000.2727

Reinitz, M. T., \& Hannigan, S. L. (2004). False memories for compound words: Role of working memory. Memory \& Cognition, 32, 463-473. doi:10.3758/BF03195839

Schacter, D. L., Koutstaal, W., Johnson, M. K., Gross, M. A., \& Angell, K. E. (1997). False recollection induced by photographs: A comparison of older and younger adults. Psychology and Aging, 12, 203-215. doi:10.1037/0882-7974.12.2.203

Shipley, W. C. (1986). Shipley Institute of Living Scale. Los Angeles, CA: Western Psychological Services.

Treisman, A., \& Schmidt, H. (1982). Illusory conjunctions in the perception of objects. Cognitive Psychology, 14, 107-141. doi:10.1016/0010-0285(82)90006-8

Tulving, E. (1985). Memory and consciousness. Canadian Psychology, 26, 1-12. doi:10.1037/h0080017

Wells, E. C., \& Pozzulo, J. D. (2006). Accuracy of eyewitnesses with a two-culprit crime: Testing a new identification procedure. Psychology, Crime \& Law, 12, 417-427. doi:10.1080/10683160500050666

Wong, J. T., Cramer, S. J., \& Gallo, D. A. (2012). Age-related reduction of the confidence-accuracy relationship in episodic memory: Effects of recollection quality and retrieval monitoring. Psychology and Aging, 27, 1053-1065. doi:10.1037/a0027686

Yonelinas, A. P. (2001). Consciousness, control, and confidence: The 3 Cs of recognition memory. Journal of Experimental Psychology: General, 130, 361-379. doi:10.1037/0096-3445.130.3.361

Yonelinas, A. P., \& Jacoby, L. L. (1995). The relation between remembering and knowing as bases for recognition: Effects of size congruency. Journal of Memory and Language, 34, 622-643. doi:10.1006/jmla.1995.1028 